\title{
Sulla fonte Z del Milione di Ramusio L'enigma di Quinsai
}

\author{
Giuseppe Mascherpa \\ (Università di Verona, Italia)
}

\begin{abstract}
The study of the two chapters of the Devisement dou monde concerning the Chinese city of Quinsai, former capital of the Song Empire and a kind of Far Eastern Venice, is crucial to understand some important dynamics of the tradition of Marco Polo's book. Indeed the synoptic reading of these chapters in the redactions $F, Z$ and $R$ - the last two far richer of information than the first - seems to confirm an old, debated hypothesis: the one according to which the Latin redaction Z and Ramusio's Italian version (mostly deriving from a lost $Z$ manuscript) would show traces of a rewriting of some parts of the book, in which some notes by Marco Polo, not used in the Franco-Italian text drafted in Genoa in 1298, would have been included.
\end{abstract}

Sommario 1 Costellazione Z. - $1.1 \mathrm{Z}$ brevior. - 1.2 La fase $\beta^{\prime \prime}$. - 2 Quinsai. - 2.1 Il testo in $Z^{\mathrm{t}}$. - 2.2 Il testo in R. - 3 Conclusioni.

Keywords Marco Polo. Devisement dou monde. Quinsai. Latin redaction Z. Ramusio's Italian version. Rewriting. Textual variants. Marco Polo's notes.

\section{Costellazione Z}

Nel corso dell'ultimo decennio, nel contesto di una rinnovata attenzione per la questione testuale del Devisement dou monde, alcuni studi hanno contribuito a meglio delineare la complessa fisionomia della redazione latina $\mathrm{Z}$, considerata a buon diritto lo snodo fondamentale della tradizione antica del libro poliano: $Z$ conserva infatti, come è noto, un gran numero di passi (da brevi integrazioni a interi paragrafi e addirittura capitoli, a versioni alternative di segmenti di testo) privi di riscontro nella restante tradizione, ${ }^{1}$ ma quasi tutti preziosi per una più precisa definizione della realtà geo-etnografica, storica e politica dell'Asia di Marco Polo.

La redazione $\mathrm{Z}$ è tramandata da un unico esemplare (Toledo, Archivo y Biblioteca Capitulares, ms. Zelada 49-20: d'ora in avanti $Z^{t}$ ), trascritto in Veneto - Venezia, o terraferma orientale - intorno alla metà del Quat-

1 Tranne che, saltuariamente, nella versione veneziana $\mathrm{V}$ e nell'epitome latina $\mathrm{L}$ (Benedetto 1928, CLXXIII-CLXXXI; Simion 2015c; Simion, Burgio 2015 [«Introduzione», § «La redazione L», a cura di Eugenio Burgio). 
trocento e purtroppo radicalmente abbreviato, soprattutto nella prima parte, grosso modo fino all'inizio del 'libro dell'India'; ${ }^{2}$ collaterale del codice toledano, ma più completo, è il perduto esemplare Z (il cosiddetto 'codice Ghisi', siglato $\mathrm{Z}^{\mathrm{g}}$ ) la cui silhouette è individuabile in filigrana al Milione di Ramusio (R), del quale costituì la fonte principale, se non per la struttura globale, senz'altro per i contenuti (le altre fonti, come è noto, furono la versione latina di Pipino [P], un libero rimaneggiamento di area veneto-lagunare [VB], e in misura molto minore la versione veneziana $\mathrm{V}$ e il compendio latino $\mathrm{L}$ ).

Gli addenda di Z, che nella maggior parte dei casi - è opinione corrente presso gli studiosi del Devisement - nulla osta a ricondurre recto tramite all'autore, o quantomeno alla sua cerchia famigliare, convinsero Luigi Foscolo Benedetto a riservare alla redazione latina una posizione altissima nell'ipotetico stemma del Devisement dou monde; lo studioso vedeva infatti in $\mathrm{Z}$ la traduzione di un testo franco-italiano molto vicino all'originale composto a Genova da Marco Polo e Rustichello da Pisa, la cui successiva, ampia sfrondatura, promossa da esigenze editoriali, avrebbe prodotto l'archetipo della restante tradizione, ${ }^{3}$ di cui il ms. français 1116 della Bibliothèque Nationale di Parigi $(F)$ sarebbe un fedele riflesso.

\subsection{Z brevior}

Questa ricostruzione, anche in ragione dell'autorevolezza di Benedetto e della monumentalità della sua sistemazione critica, ha goduto di ottima salute fino a pochi anni or sono, allorché nuove indagini sulla tradizione indiretta di $\mathrm{Z}$ in due compilazioni trecentesche, riconducibili al milieu domenicano del Veneto meridionale, hanno condotto a un sostanziale ribaltamento di prospettiva. Lo studio degli excerpta poliani contenuti nel Legendarium agiografico di Pietro Calò da Chioggia e nel Liber de introductione loquendi di Filippino da Ferrara ${ }^{4}$ (entrambi ante

2 Rispetto a $F$, Z è privo di una sessantina di capitoli, mentre altri risultano inficiati da tagli più o meno corposi (Benedetto 1928, CLXIV; Barbieri 1998, 576-8; Mascherpa 2007-08, 78-99).

3 Con l'eccezione di V e L, nei quali Benedetto e Terracini individuavano, in ragione dei loro punti di contatto con $\mathrm{Z}$ nel contesto di una sostanziale aderenza a F, l'espressione di una tappa intermedia nel processo di riduzione del Devisement originario (Benedetto 1928, CLXXXII; Terracini 1933, 409-20).

4 Sui brani del Liber de introductione loquendi («particolare raccolta di narrazioni ed exempla ordinati in otto libri, ciascuno dei quali relativo ad una 'circostanza' della vita sociale in cui un frate è chiamato a prendere la parola»: Gobbato 2015, 319-20) che Filippino desume dal Devisement dou monde è imprescindibile lo studio di Gobbato 2015. Quanto al capitolo del Devisement sulla predicazione orientale di San Tommaso Apostolo, incastonato da Pietro Calò nel suo Legendarium, l'edizione del testo è in Devos 1948, l'inquadramento ecdotico in Mascherpa 2008, ripreso in Mascherpa 2007-08, 164-71. 
1350) ha infatti rivelato come gli esemplari Z utilizzati dai compilatori fossero, almeno nei segmenti selezionati, privi degli addenda caratteristici dell'antecedente comune di $\mathrm{Z}^{\mathrm{g}} / \mathrm{Z}^{\mathrm{t}}$ (siglato $\beta^{\prime \prime}$ nel nuovo stemma del Devisement presentato in questo volume) e, se si eccettua qualche minimo incremento testuale, grosso modo coerenti con $\mathrm{F}$ nella forma e nei contenuti. ${ }^{5}$ Si considerino un paio di esempi, selezionati nell'ambito di una casistica molto varia. ${ }^{6}$

(a) Il culto di San Tommaso Apostolo nell'India sud-orientale (Mascherpa 2008)

Nella sezione del Legendarium dedicata a San Tommaso Apostolo, il capitolo di Marco Polo occupa una posizione di rilievo tra le fonti utilizzate da Pietro Calò, in quanto offre al compilatore non solo un resoconto sulla predicazione e il martirio dell'Apostolo nel Maabar e sulla persistenza di un culto locale (interconfessionale) a lui dedicato, ma anche la narrazione di un miracolo post mortem non altrimenti attestato.

Nell'estratto qui riportato emerge come l'esemplare Z utilizzato da Calò rispecchiasse le movenze di $\mathrm{F}$ e della restante tradizione $v s \mathrm{Z}^{\mathrm{t}}$, oltre che nel procedere sintattico, anche nella transizione diretta 2 a 3 senza l'inserto 2a (la coltivazione delle palme da cocco nell'area del santurario), che è proprio dei soli $\mathrm{Z}^{\mathrm{t}}$ e (si veda infra) $\mathrm{R}$.

5 L'appartenenza del Milione di Pietro Calò alla famiglia Z, che Benedetto si limitò a segnalare senza il corredo di una dimostrazione (Benedetto 1959-60, 573-5), è sancita dalla condivisione di un errore congiuntivo con il testo di Toledo: nell'episodio del martirio di San Tommaso, $\mathrm{Z}^{\mathrm{t}} \mathrm{e}$ il testo del Legendarium concordano infatti nell'affermare che il santo muore trafitto in tibiam dexteram $\left(\mathrm{Z}^{\mathfrak{t}}\right)$ /in tybia dextra (Leg.) dalla freccia scagliata da un pagano, laddove il resto della tradizione, più plausibilmente, riferisce invece di una ferita al costato: $\mathrm{F}$ destre costee, L dextrum latus, TA per le costi, V in lo ladi destro, ecc. (Simion 2017a, 26 nota 16). Nessun errore congiuntivo, ma una nutrita schiera di varianti - sia formali che sostanziali - rivela un esemplare Z anche nella fonte poliana di Filippino (Gobbato 2015, 329-55). Sulla possibilità che la versione $\mathrm{V}$ dipenda da uno $\mathrm{Z}$ con le stesse caratteristiche di quelli utilizzati dai domenicani si vedano le osservazioni di Samuela Simion raccolte e commentate in Simion 2011 e, più recentemente, in Simion 2015c. Pare uno Z di questo tipo anche quello utilizzato, probabilmente per contaminazione, nella stesura dell'epitome L.

6 Negli esempi, in carattere sottolineato le lezioni che accomunano tutti i testimoni $Z$ vs $F$, in corsivo quelle riconducibili al solo antecedente comune di $\mathrm{Z}^{\mathrm{t}} / \mathrm{Z}^{\mathrm{g}}\left(\beta^{\prime \prime \prime}\right)$. 


\section{$Z^{\mathrm{t}} 109$ 1-15}

1. Corpus quidem Beati Thome apostoli est in provincia Maabar in quadam civitate parva, in qua sunt pauci mercatores et homines, neque illuc veniunt quia ibi sunt pauca mercimonia que illinc possi<nst extrahi, et etiam locus multum devius est.

2. Bene verum est quod multi christiani et saraceni illuc veniunt propter devotionem: nam saraceni illius contrate habent magnam devotionem in ipso; dicunt enim quod fuit saracenus et quod magnus propheta est, et nuncupant eum avarium, quod est dicere 'sanctus homo'.

\section{2a. Christiani qui ecclesiam custodiu[nt] multas habent arbores, que vinum faciunt et que nuces pharaonis producunt nam de una nuce pasceretur unus homo cibo et potu [...] pro qualibet arborum istarum solvunt christiani uni ex quatuor fratribus regibus in provincia Maabar in quolibet mense groso uno.}
3. Et dicemus vobis de mirabilibus que ibi sunt. Noveritis itaque quod christiani qui illuc propter devotionem accedunt accipiunt de terra ubi fuit mortuus sanctus thomas et illam terram in eorum patriam perportant et dant ad potandum de ista terra pacientibus febres tercianas vel quartanas et statim cum eger potaverit liberatus est. Et hoc accidit omnibus egris potantibus hanc terram; terra quidem rubea est. Et dominus Marcus de hac terra secum portavit Venecijs et multos liberavit cum ipsa.

\section{Legendarium}

1. Est igitur corpus eius in quadam civitate parva in qua sunt pauci mercatores et homines, neque illuc veniunt quia ibi non sunt mercimonia que inde possint extrahi, et est locus multum devius.

2. Multi autem christiani et saraceni illuc veniunt propter devotionem in eum, et dicunt quod fuit saracenus, in hoc mencientes, quia Thomas apostolus iudeus fuit, et nominant eum avarion, id est bonum hominem.

\section{F 175 2-4}

1. Le cors meisser Saint Thomeu le apostres est en la provence de Meabar, en une petite ville; car ne a gueires homes, ne mercaant ne $\mathrm{i}$ vienent por ce que n'i a mercandies que bien en peust traire et encore que le leu est mout desviables.

2. Bien est il voir que maint cristiens et mant saraçin hi vienent en perlinajes: car je voç di que le saraçin de celle contree hi ont grant foi et dient qu'il fui saraçin, et dient qu'el est profit grant et l'apellent avarian, que vaut a dire saint home.

\begin{abstract}
3. Christiani autem quui illuc propter devotionem accedunt, accipiunt de terra ubi mortuus fuit sanctus apostolus, et illam in suam patriam portant, et dant ad potandum de ista terra cuicumque pacienti febres quartanas vel tercianas vel alias. Et statim cum eger potaverit liberatus est. Et hoc accidit omnibus egris potantibus de hac terra, que est rubea. Et dominus Marchus prefatus portavit secum de terra ista Venecias et multos liberavit cum ipsa.
\end{abstract}

\begin{abstract}
3. E si sachiés que il hi a tel mervoie com je vos conterai: or saquiés que les cristienç, que vont la en pelegrinajes, prenne«n`t de la tere dou leu, la ou le saint cors fou mort, e celle terre aportent en le lor contree, e donent de ceste une pou a boir au malaide quant ausse fevre quartaine ou tersaine ou ceste tiel fevre; et tant tost que le malaide l'a beue, el est guaris. Et ce avint a tuit celz amalaides que celle terre boivent, e sachiés que'elle est terre roge.
\end{abstract}

Ramusio desume l'intero episodio da P ma ne integra il dettato con la pericope sulle palme da cocco, che in $\mathrm{Z}^{\mathrm{g}}$ doveva leggersi in una forma molto vicina alla lezione toledana: ${ }^{7}$

7 Ramusio taglia però la descrizione del frutto, già utilizzata nel capitolo su Samara (III 13 8) per integrare la lezione di VB (136 11). 
... Et molti altri miracoli tutto il giorno si veggono, per intercessione di questo beato apostolo. I christiani che custodiscono detta chiesa hanno molti arbori che fanno le noci d'India che habbiamo scritto di sopra, quali li danno il vivere, et pagano ad un di questi re fratelli un grosso ogni mese per arbore. (R III 20 65-6)

(b) Le palme da sago nel regno di Fansur (Gobbato 2015, 352-3)

La digressione 'meravigliosa' sulla coltivazione (a tutt'oggi fiorente) della palma di sago a Sumatra fa parte dell'eterogeneo drappello di tessere poliane incluse nel Liber de introductione loquendi di Filippino da Ferrara. Di nuovo, come nel caso di Pietro Calò, ci si imbatte in un esemplare Z aderente al dettato di $F$ nella forma e nei contenuti: ${ }^{8}$ manca infatti, nella fonte $\mathrm{Z}$ di Filippino, il minuto resoconto sull'estrazione e lavorazione della 'farina' che invece caratterizza $\beta$ '".

\section{$Z^{\mathrm{t}} 103$ 6-13}

...habent enim maneriem unam arborum que sunt multum grose et longe, et earum lignum est circumcirca forte per tres digittos grossum, et tota medula interior est farina, [...] et farina emundata in fundo remanet comprehensa, et tunc conditur et fiunt ex ea lagana et diverse epule que de pasta fiunt, que sunt valde bone, et Dominus Marcus multociens hoc probavit

\section{R III 16 4-6}

...hanno una sorte di arbori grossi et lunghi, alli quali levatali la prima scorza, che è sottile, si trova poi il suo legno grosso intorno intorno per tre dita, et tutta la midolla di dentro è farina come quella del caruol [...] et la farina purgata et mondata che rimane si adopra, et fansi di quella lasagne et diverse vivande di pasta, delle qual ne ha mangiato piú volte il detto messer Marco

\section{Liber I 20 2-4}

...est una generatio arborum que habent corticem subtilem, et sub cortice sunt plene farina, et faciunt multa comestibilia de pasta que sunt valde bona. Et dominus Marcus hoc probavit multociens.

\begin{abstract}
F 1696
...il ont une mainere d'arbres qe mout sont groses e grant. E cesti arbres sunt tuit plein dedans de farine; qe sachiés qe cesti arbres ont mout soutil escorces, e tuit dedens est farine, et ne font meint mengier de paste qe mout sunt buen a mangier, car je voç di qe nos meesme les provammes aseç, car nos en menuiames plusors foies
\end{abstract}

\subsection{La fase $\beta^{\prime \prime \prime}$}

Gli esempi (a) e (b) dimostrano dunque l'esistenza e la circolazione di quello che si potrebbe definire uno ' $\mathrm{Z}$ brevior': ovvero una prima versione latina, messa insieme nella prima metà del Trecento, di un testimone franco-italiano non distante da F ma qualche punto più ricco. Sullo sche-

8 Meno che nello sviluppo «...qe mout sont groses e grant. E cesti arbres sunt tuit plein dedans de farine; qe sachiés qe cesti arbres...», mancante in tutti i testimoni del trattato di Filippino: può trattarsi di lacuna per saut du même au même (su arbores/arborum?) prodottasi nell'archetipo del Liber o già presente nella fonte del domenicano, oppure della semplice sfrondatura di un passo oggettivamente ridondante. 
letro di questa traduzione si sarebbe poi compiuto un ulteriore processo rielaborativo, volto essenzialmente all'espansione (anche considerevole) del testo di partenza, sia tramite la riscrittura approfondita di segmenti testuali già dati, che con l'innesto di brani - descrittivi e narrativi - di dimensioni variabili e completamente nuovi. Prodotto di questa fase redazionale è $\beta^{\prime \prime \prime}$, la cui fisionomia complessiva si può ricavare virtualmente dalla triangolazione tra $Z^{t}, R$ (laddove rifletta $Z^{g}$ ) e F.

Quello che emerge dalla sinossi, in coerenza con le osservazioni di Benedetto, è prima di tutto lo scarto qualitativo che separa $\mathrm{Z}^{\mathrm{g}}$ e $\mathrm{Z}^{\mathrm{t}}$ sotto il profilo dell'integrità testuale in corrispondenza dei primi due libri di $\mathrm{R}$, dove la versione ramusiana consente la ricostruzione della silhouette di $\beta^{\prime \prime \prime}$, emendando la lezione aspramente ridotta o del tutto mancante del Toledano. Con il terzo libro si innesta invece il movimento contrario: è la lezione di $\mathrm{Z}^{\mathrm{t}}$, da un certo punto in avanti sostanzialmente completa, a restituire il testo di $\beta^{\prime \prime \prime}$, mentre Ramusio ricorre più spesso alle altre fonti $\mathrm{e}$, quando utilizza $\mathrm{Z}^{\mathrm{g}}$, non esita a sunteggiarlo, sia nei passi in comune con la tradizione che negli addenda (o forse $\mathrm{Z}^{\mathrm{g}}$, con situazione speculare a quella del Toledano, presentava in questa sezione un testo lacunoso).

In qualche luogo, la sinossi $Z^{\mathrm{t}}-\mathrm{R}-\mathrm{F}$ serve altresì a evidenziare come nella 'fase Z' non abbiano agito soltanto dinamiche espansive, ma anche, contestualmente ad esse, strategie di riorganizzazione testuale (rimescolamento di tessere; contrazione/accorpamento) tipiche di un po' tutta la tradizione antica del Devisement. Emergono in particolare casi di

a. interventi sulla dispositio delle pericopi: ad esempio, nei capitoli dedicati alla pianura tra Qamadin e Hormuz (F XXXV-XXXVI; R I 14-16; Z 13-14), nei quali $\beta$ "' presenta una scaletta alternativa, complessivamente più razionale, del materiale corografico;

b. riduzione del dettato con accorpamento di capitoli e tagli di varia entità: ad esempio nel racconto del miracolo di Baghdad, sfrondato di molti pleonasmi (F XXV-XXVIII; R I 8 11-29; Z 7); nella sezione dedicata al Vecchio della Montagna (F XL-XLII; R I 21; Zt 17), dove vengono eliminate le ridondanze della parte centrale (corrispondente, all'incirca, al segmento di F XLI 6-XLII 7); nel capitolo su Samarcanda (R I 30 4-7; F LI 5-17; Z 26 1), che in $\beta^{\prime \prime \prime}$ mancava probabilmente della narrazione del miracolo di San Giovanni Battista. In tutti e tre i casi - oltre che in molti altri, anche minuti - il fatto che Ramusio ricorra a P o VB nei punti esatti in cui $\mathrm{Z}^{\mathrm{t}}$ si presenta abbreviato o mancante induce a credere che anche $\mathrm{Z}^{\mathrm{g}}$ recasse, nei passi in questione, un testo scorciato. ${ }^{9}$

9 Per ragioni di sintesi, si rimanda ai commenti ad loca contenuti in Simion, Burgio 2015. 
Un ultimo, ma non meno rilevante aspetto sul quale la sinossi invita a riflettere è la questione degli addenda singolari di $\mathrm{R} \mathrm{e} \mathrm{Z}^{\mathrm{t}}$, quelli che, attestati soltanto nell'uno o nell'altro testimone, non sono riconducibili a $\beta$ '"' per via stemmatica. ${ }^{10}$ In molti casi questi ajouts hanno l'aspetto di glosse esegetiche o di note erudite alla portata di qualunque copista o rielaboratore. ${ }^{11}$ Altrove invece si tratta invece di brani genuini nei contenuti (di 'sapore poliano', si potrebbe dire) e talora così lunghi e articolati da costituire unità testuali autonome nel mosaico multiforme di Z. Oltre all'ampia casistica offerta, sia per $\mathrm{Z}^{\mathrm{t}}$ che per R, dal capitolo su Quinsai (cf. infra, § 2), si ricordano, tra i brani singolari di R, il capitolo su un monastero persiano intitolato all'eremita Barsauma (I 10), la descrizione degli effetti devastanti del caldo di Hormuz (I 15 8-10), il breve excursus - certamente genuino ${ }^{12}$ - sui tatuatori dello Yunnan (I 18 4; cf. infra, es. (a)); tra gli ancora più numerosi unica di $\mathrm{Z}^{\mathrm{t}}$, invece, il capitolo corografico sullo Iuguristan (33), l'aneddoto sulle facoltà psicotrope della terra del Kirman (12 4-11: es. (b)), o quello sulle stravaganti pratiche divinatorie dei marinai indiani (91 30-40).

In linea di massima, mancando prove testuali cogenti, non v'è ragione di dubitare che tutti questi addenda fossero effettivamente presenti in $\beta^{\prime \prime \prime}$, e che la loro assenza - a seconda - in $\mathrm{Z}^{\mathrm{t}}$ o $\mathrm{R}$ sia da imputare, per il primo, ai tagli del copista-riduttore, per il secondo, alle scelte editoriali di Ramusio, oppure a una campagna di sfoltimento messa in atto dai rispettivi antecedenti: dinamiche dietro alle quali non è sempre possibile individuare un disegno coerente. Ci si può tuttavia imbattere (a) in casi in cui $\mathrm{R}\left(<\mathrm{Z}^{\mathrm{g}}\right)$ presenta un addendum singolare laddove $Z^{t}$ ricalca nella struttura e nei contenuti il testo della tradizione $\left(\mathrm{Z}^{\mathrm{t}}=\mathrm{F}\right.$ vs $\left.\mathrm{Z}^{\mathrm{g}}\right)$; (b) oppure, specularmente, in tessere singolari di $Z^{\mathrm{t}}$ laddove $\mathrm{R}\left(<\mathrm{Z}^{\mathrm{g}}\right)$ risulta allineato a $\mathrm{F}\left(\mathrm{Z}^{\mathrm{g}}=\mathrm{F}\right.$ vs $\left.\mathrm{Z}^{\mathrm{t}}\right)$.

10 Onde evitare falsi positivi, si circoscrive l'indagine sugli addenda singolari alle sole sezioni in cui $\mathrm{Z}^{\mathrm{t}}$ non sia drasticamente scorciato.

11 Qualche esempio: R I 8: «[il ciabattino] immediate con una delle stecche che adoprava in bottega si cavò l'occhio destro; la qual cosa dimostrò manifestamente la grandezza della sua constante fede»; R III 43 8: «Ha un castello molto forte, che tiene a modo di dir serrato il colfo et il mare, perché discopre tutte le navi da ogni tempo che passano»; $\mathrm{Z}^{\mathrm{t}}$ 9: «diem miraculi huius Christiani Nestorini et Iacopiti solempniter celebrant, continue in vigilia ieiunantes. Nota quod christiani, armeni, nestorini et iacopiti diferunt in certis articulis, ymo ex hoc unus alium repudiat et aborret»; $\mathrm{Z}^{\mathrm{t}} 101$ 10: «Na<m» si substantia aliqua remaneret, illa procrearet vermes; et vermes deficiente esca morirentur; et de obitu istorum vermium dicunt ipsi quod anima defuncti pecatum magnum haberet, videlicet quod tot anime ex substantia sua orte perirent» (cf. le schede di Burgio e Mascherpa ad loca in Simion, Burgio 2015).

12 L'informazione di R aderisce perfettamente al dato etnografico: cf. Yule, Cordier 1903, 2: 128 . 
(a)

\section{R || 41 3-5}

Gli huomini et le donne di questa provincia usano di portare li denti coperti d'una sottil lametta d'oro, fatta molto maestrevolmente a similitudine di denti, che li coprono, et vi sta di continuo

Gli huomini si fanno anchor a torno le braccia et le gambe a modo di una lista o vero cinta, con punti neri, designata in questo modo: hanno cinque agucchie tutte legate insieme, et con quelle si pungono talmente la carne che vi esce il sangue, et poi vi mettono sopra una tintura nera, che mai piú si puol cancellare; et reputano per cosa nobile et bella haver questa tal lista di punti neri.

Et non attendono ad altro se non a cavalcare et andare alla caccia et uccellare...

(b)

\section{$Z^{\mathrm{t}}$ 12-13 1}

Chermam quoddam regnum est in confinibus Persie versus levantem, quod heredes antiquitus succedebant. In isto regno lapides oriuntur nomine turchiese, qui fodiuntur in venis montium; inveniuntur etiam in montibus illis vene calibis et andaniqui in maxima quantitate. In hyeme tam intensum frigos $h$ rabsetur, quod vix homo potest evadere continue portando multas vestes et peles.

\section{$Z^{t} 58$ 3-4}

Gentes ipsius dentes habent deauratos, videlicet quod omnes dentes sunt coperti auro: nam fieri faciunt in similitudinem dentium unam formam de auro, cum qua dentes coperiunt.

Homines itaque, secundum eorum consuetudinem, milites sunt omnes, et ad nil aliud intendunt quam ad aucupationes, venationes...

\section{R I 13-14 1}

Chiermain è un regno ne' confini della Persia verso levante, il qual anticamente andava de herede in herede, ma dopo che 'I Tartaro lo soggiogò al suo dominio non succedettero gli heredi, anzi il Tartaro vi manda signore secondo il voler suo. In detto regno nascono le pietre che si chiamano turchese, quali si cavano nelle vene de' monti; si trovano anchora in quelli vene di azzaio et andanico in grandissima quantità. [...] nel tempo dell'inverno vi è cosí gran freddo, che appena l'huomo si può riparare portando continovamente molte vesti et pelli.

Dapoi la discesa di questo luogo per le dette due giornate si trova una gran pianura

\section{F 119 4-5}

Les jens ont tous les dens d'or, ce est qe chascun dens est covert d'or, car il font une forme d'or faite a la mainere de seç dens et couvrent les dens desout come celz desovre; et ce font les homes et nen les dames.

Et les homes sunt tuit chevalers, selonc lor usance, et ne font rien for qe aler en host et aler chaçant et oselant

\section{F 34-35 2}

Crerman est un rengne en Perse mesme et ansiene (ment) so seingnore l'oit por hereditajes; mes, puis que le Tartar le conquistrent, ne vait pas la segnorie por hereditajes, mes hi mande le Tartar celui sire qu'il vult. En cest regne naisent les pieres que l'en apele torchoise, et hi ni a en grant habundance, car il les trevent en les montagnes, car il le escavent dedens la roche. Et encore ont vene d'acier et d'ondanique assés. [...] ha si grant froit de yver que a poine eschanpe l'en portant aséç dras et aséç pannes.

Et quant l'en ha descendu celle deus jorné que je voç ai dit, adonc treve une grandisme plaingne... 
Gli esempi mostrati sembrano aprire alla possibilità che qualcuna delle aggiunte più significative di $\mathrm{Z}$ sia stata accolta a testo non all'altezza di $\beta^{\prime \prime \prime}$, bensì dei rispettivi antigrafi di $Z^{\mathrm{t}} \mathrm{e} R\left(\mathrm{Z}^{\mathrm{g}}\right)$. Il che rivelerebbe, almeno per alcune parti del libro, una 'fase $Z$ ' testualmente irrequieta e attiva fino ai piani più bassi della sua tradizione, nella quale anche le ultime diramazioni dello stemma sarebbero state singolarmente permeabili all'innesto di materiali nuovi - se non proprio riconducibili all'autore, almeno autentici nei contenuti e nella forma coerenti con l'habitus poliano.

Del resto, numerosi indizi - la presenza di addenda genuini, il marcato radicamento lagunare ${ }^{13} \mathrm{e}$ in particolare la circolazione entro il milieu domenicano della città di Venezia ${ }^{14}$ - suggeriscono che la redazione $\mathrm{Z}$ sia stata effettivamente prodotta in un contesto prossimo alla cerchia familiare dell'autore, e che la sua mouvance ( $\mathrm{Z}$ brevior $>\beta^{\prime \prime \prime}>$ innesti singularia in $\left.\mathrm{Z}^{\mathrm{g}} / \mathrm{Z}^{\mathrm{t}}\right)$ rappresenti in qualche modo il 'processo evolutivo' del testo di Marco Polo.

Le più consistenti pezze d'appoggio a questa ricostruzione risiedono nelle dinamiche testuali che coinvolgono, in $\mathrm{R}$ e $\mathrm{Z}^{\mathrm{t}}$, la monografia sulla città di Quinsai, la cui complessità rende indispensabile una trattazione autonoma.

\section{Quinsai ${ }^{15}$}

Tra XIII e XIV secolo la città di Quinsai (oggi Hang-zhou), antica e splendida capitale dell'impero Song conquistata dai Mongoli nel 1276, veniva celebrata dai viaggiatori occidentali come una delle più straordinarie meraviglie urbane del Levante. Percorsa da una fitta rete di canali e affacciata su un grande lago, Quinsai godeva di un clima mite e di una posizione strategica. La sua particolare topografia, insieme alle vie brulicanti di vita,

13 A quest'area puntano quasi esclusivamente gli indizi offerti dalla geografia della tradizione: $\mathrm{Z}^{\mathrm{t}}$ è localizzabile, sulla base della filigrana, a Venezia o tutt'al più nel Veneto orientale (Mascherpa 2007-08, 17-18); di ambito veneziano sono le redazioni L, V e VB, in varia misura compromesse con la 'fase Z' (Burgio, Mascherpa 2007; Simion, Burgio 2015). Dal convento dei Santi Giovanni e Paolo transitarono - uno come studente, l'altro come priore - Filippino da Ferrara nel 1307 e Pietro Calò nel 1328 (Gobbato 2015, 357); a San Michele di Murano il camaldolese fra Mauro, attorno al 1450, utilizzò un esemplare Z come fonte per la compilazione della sua Mappa mundi (Cattaneo 2005b; Burgio 2009). A Venezia e su materiali principalmente veneziani lavora, infine, Ramusio.

14 Sul rapporto tra Marco Polo e i domenicani del convento dei SS. Giovanni e Paolo di Venezia si veda ora Gobbato 2015, 357-60.

15 In questo paragrafo rifondo parte dei contenuti di un mio recente contributo, in corso di stampa negli atti del convegno internazionale Predicatori, mercanti, pellegrini. L'Occidente medievale e lo sguardo letterario sull'Altro tra l'Europa e il Levante (Sassari, 11-12 maggio 2016). 
alle piazze invase dai mercati e ai palazzi signorili, deve avere restituito agli occhi di Marco Polo l'immagine di una splendida Venezia d'Oriente, la cui descrizione si sviluppa nell'ampia e articolata monografia contenuta nei capitoli 151 e 152 di F. ${ }^{16}$ La sostanza testuale e l'ordine espositivo della redazione franco-italiana sono ribaditi senza variazioni degne di nota in quasi tutti i testimoni della tradizione antica, ${ }^{17}$ meno che in $\mathrm{Z}^{\mathrm{t}} \mathrm{e}$ - soprattutto - in R, la cui versione del trattato sulla 'città del cielo' (e in particolare del primo dei due capitoli ad essa dedicati) risulta profondamente rivoluzionata. ${ }^{18}$

\subsection{Il testo in $\mathrm{Z}^{\mathrm{t}}$}

Nel codice Toledano i capitoli su Quinsai (85-86) presentano una dispositio degli argomenti sostanzialmente identica a quella di F, fatta eccezione per l'inversione di due excursus etnografici adiacenti dedicati alle pratiche astrologiche e alle consuetudini funebri del Mangi (cf. tav. 1). Differenze sostanziose si notano invece sul piano dei contenuti, dove, al netto dei tagli prodotti dall'azione indiscriminata del riduttore - sia nel corpo del capitolo che in fine, dove un etcetera tronca bruscamente il flusso testuale $^{19}$ - il testo toledano risulta in più punti arricchito dall'inserzione di ajouts di dimensioni variabili del tutto sconosciuti alla restante tradizione, tranne che, in qualche caso, a R (che ne condivide alcuni ma non altri): si tratta di aggiunte brevi innestate su porzioni testuali già date, allo scopo di precisarle (a1) e, nel caso, rettificarle (a2), o di brani nuovi molto estesi e del tutto autonomi rispetto al testo della tradizione (es. [b1], [b2]), principalmente di carattere etnografico e storico, con digressioni legate alla conquista mongola della città, di cui Marco Polo fu testimone diretto.

16 F 151 (rubr.): «Ci devise de la noble cité de Quinsai»; F 152 (rubr.): «Ci devise de la grant rende que le Gran Kaan a de Qinsay».

17 Quanto all'ordine degli argomenti, le innovazioni più interessanti si riscontrano nel 'Milione veneto' VA (114): si tratta però, con ogni probabilità, di interventi di ristrutturazione operati da un rimaneggiatore ai piani bassi dello stemma, giacché il testo di Pipino, dipendente da un esemplare VA più vicino all'archetipo, presenta la stessa struttura di F. Alcune singolarità strutturali occorrono anche in VB 114, che in un luogo del testo presenta addirittura un addendum senza riscontri nella tradizione (cf. Gennari 2008-09, 161, § 17 e la nota di A. Andreose a R II 6863 in Simion, Burgio 2015). Mancano infine alcune pericopi in L, V, per il resto sostanzialmente allineati a $\mathrm{F}$.

18 Struttura e contenuti del capitolo sono riassunti nelle tavole sinottiche (F-Z; F-R) poste in appendice.

19 Gli etcetera di $\mathrm{Z}^{\mathrm{t}}$ troncano pericope e capitolo anche nei capp. 31, 45-7, 79, 100; interrompono pericopi interne nei capp. 59 e 90. 
(a1) Il materiale dei ponti ${ }^{20}$

\section{F 1517}

Il se contenoit tuit primermant ‘ques la cité de Quinsai gir'environ .C. miles et ha .XII ${ }^{\mathrm{m}}$. pont de pieres

\section{$Z^{\mathrm{t}} 857$}

Primo quidem continebatur in eis quod civitas Qinsay in circuitu suo girat miliaria centum tere, et habet duodecim milia pontium, pro maiori parte de lapidibus, quia aliqui de legnamine sunt constructi

\section{(a2) Norme di Khubilai in merito ad arti e mestieri}

\section{F 1519}

Et si voç di qu'il estoit establi por lor roi qe cascun doie faire les ars de son pere; et, se il avesse. $\mathrm{C}^{\mathrm{m}}$. besanç, ne poroit fair autre ars qe sun pere avoit fait.

\section{$Z^{\mathrm{t}} 85$ 16-18}

Sanccitum erat itaque per Factur, regem dicte civitatis et provincie, tempore quo regnabat, quod quilibet deberet exercere artem sui patris; et si quis haberet centum milia bysantiorum, oportebat ipsum artem patris exercere. Non equidem credatis quod ipsos oporteret propris manibus laborare, sed homines retinere, ut dictum est superius, ad dictam artem exercendam. Sed ad hoc per Magnum Dominum minime compelluntur: nam, si homo artifex in tantas creverit divicias quod artem suam derelinquere possit et vellit, per nullum ad artis exercitium amplius coartatur...

\section{(b1) La pratica del suicidio-vendetta ${ }^{21}$}

\section{$Z^{\mathrm{t}} 85$ 43-46}

Sunt itaque homines provincie Mançi pre alijs gentibus iracundi, et pre ira et dolore ipsimet quam plures aliqui se occidunt. Nam si accidit quod ex ipsis aliquis alteri alicui $i<m>$ primat alapam vel ipsum decapillet aut aliquam sibi inferat iniuriam et gravamen, et iniuriator sit tan potens et magnus quod ille sit impotens ad vindictam, pre nimio dolore passus iniuriam se de nocte ad portam iniuriatoris suspendet et morietur, hoc sibi faciens ad maius vituperium et contemptum...

20 In corsivo, qui e infra, gli addenda.

21 Su questa pratica estrema di rivalsa sociale si rimanda a Barbieri (1998, 214-5 nota 4). 
(b2) L'assedio mongolo e il drago del fiume Qiantang

\section{$Z^{\mathrm{t}} 8562-66$}

Dicimus de quodam miro quod accidit cum Bayan erat in obsidione civitatis istius, videlicet quod cum rex Facfur a facie eius fugit, multitudo gentium ipsius civitatis in navigio fugiebat per quoddam maximum flumen, latum et profundum, quod transit ab uno latere civitatis. Et dum sic per flumen istud fugerent, ipso instanti fuit aqua totaliter desiccatum, ita quod Bayan, hoc inteligens, adivit partem illam, et omnes fugientes ad civitatem redire coegit. Et inventus fuit piscis unus iacens in sicco per transversum alveum fluminis, qui erat quid mirabile ad videndum : nam longitudinis erat bene pasuum centum, grossitudo vero minime longitudini respondebat. Pilosus quidem erat totus, et multi del ipso comederunt, et multi ex ipsis perierunt. Et dominus Marcus, ut dicit, capud illius piscis propriis oculis vidit in templo quoddam ydolorum, etcetera.

\subsection{Il testo in $\mathrm{R}$}

Nei capitoli su Quinsai (R II 68-69), come nel resto della compilazione ramusiana, la fonte più spesso riconoscibile è $\mathrm{Z}^{\mathrm{g}}{ }^{22}$ lo dimostrano le numerose e stringenti coincidenze testuali tra $\mathrm{R} \mathrm{e} \mathrm{Z}^{\mathrm{t}}$ sia in alcuni addenda (es. a1, a2) che nei brani comuni a tutta la tradizione (b1, b2), oltre alla stretta aderenza di $\mathrm{R}$ a $\mathrm{F}$ nei luoghi in cui il Toledano è lacunoso (c): ${ }^{23}$

\section{(a1)}

\section{$Z^{\mathrm{t}} 85$ 16-17}

Sanccitum erat itaque per Factur regem dicte civitatis et provincie, tempore quo regnabat, quod quilibet deberet exercere artem sui patris, et si quis haberet centum milia bysantiorum, oportebat ipsum artem patris exercere. Non equidem credatis quod ipsos propris manibus laborare, sed homines retinere, ut dictum est superius, ad dictam exercendum.

\section{R II 6835}

Et anchor che per li re antichi fosse ordinato per legge che ciascun abitante fosse obligato ad esercitare l'arte del padre, nondimeno, come diventino ricchi, gli è permesso di non lavorar piú con le proprie mani, ma ben erano obligati di tenere la bottega, e uomini che v'esercitassino l'arte paterna.

22 L'indagine microscopica condotta da Alvise Andreose su R II 68 (reperibile ad locum in Simion, Burgio 2015) permette di ipotizzare che $Z^{g}$ sia stato il testo-base per i §§ 30,42, 47-9, 55, 58, 65-70, 91-2; P per i §§ 4, 9, 56-7, 89-90; VB per i §§ 1-3, 36, 63-4, 74. Più fonti concorrono paritariamente alla costituzione del testo nei §§ $32,33,35,53-4,75(Z+P), 34$, $43(\mathrm{P}+\mathrm{VB}), 87(\mathrm{Z}+\mathrm{P}+$ minima da VB), mentre un modello non identificabile (sull'ipotesi che si tratti sempre di $\mathrm{Z}^{\mathrm{g}} \mathrm{cf}$. infra) dà forma alla porzione più consistente del capitolo (§§ 5-8, 10-29, 31, 37-41, 44-6, 50-2, 59-62, 71-3, 76-86, 88). R II 69, invece, è interamente costruito su $\mathrm{Z}^{\mathrm{g}}$, con pochi e minimi/poco significativi apporti da P e VB.

23 In corsivo gli addenda. 
(a2)

\section{$Z^{t} 8535$}

...in aliquibus sunt mille homines, in aliquibus .$X^{m}$., in aliquibus . $X X^{m}$., in aliquibus . $X X X^{m}$., plures et pauciores secundum condicionem terrarum et potenciam earum. (?)

(b1)

\section{$Z^{\mathrm{t}} 8532$}

Item per totam civitatem istam solubrice, idest saleçate, sunt omnes vie et strate de lapidibus et lateribus, et similiter saleçate sunt omnes vie et strate provincie Mançi.

(b2)

\section{Zt 86 1-2}

Ad presens dicere volumus et narare immensam quantitatem reddituum quos Magnus Dominus ab ista civitate percipit et $a b$ aliis terris que civitati isti respondent, que sunt pars nona, sive regnum nonum, provincie Ma`n >çi. Et dicemus primo de sale, quia plus quantum ad reditum valet. (?)

\section{(c)}

\section{R II 6843}

... et quando alcuno vuol celebrar nozze, o vero far qualche solenne convito, va ad uno di questi palazzi, dove gli vien dato tutto quello che per questo effetto gli è necessario, cioè vasellami, tovaglie, mantili et cadauna altra cosa. (?)

\section{R II 6869}

...in alcune [città] vi saranno mille huomini, in altre diecimila o vero ventimila, secondo che 'I giudicherà che quella città sia piú et manco potente. (?)

\section{R II 6849}

Et prima è da sapere che tutte le strade di Quinsai sono saleggiate di pietre e di mattoni, e similmente sono saleggiate tutte le vie e strade che corrono per ogni canto della provincia di Mangi. (?)

\section{R II 691}

Hor parliamo alquanto della entrada che ha il Gran Can della città di Quinsai et dell'altre a quella adherenti: il Gran Can riceve da detta città et dall'altre che a quella rispondono, che è la nona parte o vero il nono regno di Mangi; et prima del sale, che val piú quanto alla rendita. (?)

\section{F 15112}

Et quant l'en vuelt faire noses ou convit, il vunt a ceste palais et la font lor noses et lor feste, et iluec treuvent toutes les aparoillement qe beçongne au convive, ce est de vaicellemant et de tailleor et d'escueles. (?)

Se R II 69 riproduce la lezione di $\mathrm{Z}^{\mathrm{t}}$ in maniera pedissequa, più complessa è l'interpretazione di R II 68, che rimane l'episodio più enigmatico dell'intera compilazione ramusiana: rispetto all'intera tradizione, infatti, il capitolo si 
caratterizza per la presenza di numerosi ed estesi unica, perlopiù di taglio descrittivo (approfondimenti sulla topografia della città, cenni di architettura ed edilizia, spaccati di vita cittadina), la cui inserzione in vari punti del testo produce modifiche radicali nell'ordine degli argomenti (cf. tav. 2).

Le aggiunte singolari di $\mathrm{R}$ offrono informazioni dettagliatissime e, laddove le si possa verificare, del tutto coerenti con la realtà storica, geografica e sociale della Quinsai del secondo Duecento. Sono per esempio attendibili, a dispetto di quanto è stato spesso rilevato, i passi esclusivi che Ramusio dedica ai bagni pubblici di Quinsai: il testo di R pone l'attenzione sulla consuetudine degli abitanti della città di lavarsi con acqua fredda, mentre in F 15119 e nel resto della tradizione - $\mathrm{Z}^{\mathrm{t}}$ purtroppo qui è compendioso - si fa riferimento ai soli bagni caldi. La nota sui bagni freddi, tacciata di inesattezza da Yule («This is in contradiction with the notorious Chinese horror of cold water for any purpose», Yule, Cordier 1903, 2: 246) e per questo addotta a prova della scarsa attendibilità di alcune aggiunte di R, è invece accolta come genuina da Gernet e trova qualche pezza d'appoggio - lo segnala Schafer - nella documentazione del periodo Ming (Schafer 1956, 70-1; Gernet 1959, 135-6). Corrette sono anche le informazioni sui ponti carrabili (a) e sulle carrozze da diporto (b), peraltro confermate - con qualche sorprendente coincidenza testuale - da un paio di passi del Mengliang lu: $:^{24}$

\section{R II 68}

Hor sopra questa strada di continuo si veggono andar su et giú alcune carrette lunghe, coperte et acconcie con panni et cussini di seda, sopra le quali vi possono stare sei persone, et vengono tolte ogni giorno da huomini et donne che vogliono andare a solazzo: et si veggono tutt'hora infinite di queste carrette andar a lungo di detta strada per il mezzo di quella, et se ne vanno a' giardini

\section{R II 68}

[I ponti] che sono fatti sopra i canali maestri et la strada principale sono stà voltati tanto alti [...] et nondimeno vi passano sopra carrette et cavalli, talmente sono accommodate piane le strade con l'altezza.

\section{Mengliang lu, cap. 2 (trad. Moule)}

The carriages and horses going and coming in vast numbers block the gates of the capital. Those who picnic in the suburbs go to famous gardens, beautiful parks, or places where there are strange flowers and rare trees [...] At this time (very early in the morning) the processioni is still going on.

\section{Mengliang lu, cap. 7 (trad. Moule)}

The Chung-an and Kuan bridges are both level with the Imperial Street, and so at the four quarter-days the Emperor's coaches pass by way of these two bridges. 
Di queste informazioni peculiari di R, almeno due - la grande quantità di viveri spacciata nei mercati di Quinsai (§§ 29-30) e le gite lacustri (458 ) - sono certamente riconducibili a $\beta^{\prime \prime \prime}$, stante la sopravvivenza di qualche loro traccia (rispettivamente ai §§ 60-1 e 26-7) nel compendioso Toledano. Ma anche ammesso di voler attribuire tutti i singularia di R alla 'fase Z', giustificando la loro assenza in $\mathrm{Z}^{\mathrm{t}}$ con le riduzioni operate dallo scriba, restano da sciogliere due nodi complicati: (1) la disposizione eccentrica delle tessere di $\mathrm{R}$, diversa da quella di $\mathrm{Z}^{\mathrm{t}}$ ( = F et alii) e al contempo difficilmente attribuibile all'iniziativa dell'editore; ${ }^{25}$ (2) la mancata corrispondenza tra $\mathrm{R}$ e $\mathrm{Z}^{\mathrm{t}}$ in tre passi (qui di seguito alle lettere $\mathrm{a}, \mathrm{b}, \mathrm{c}$ ) nei quali il Toledano, non abbreviato, procede di pari passo con $\mathrm{F}$ e la tradizione, mentre $\mathrm{R}$ testimonia vere e proprie versioni alternative.

(a) La descrizione dei ponti ${ }^{26}$

\section{F 1517 \\ $Z^{t} 85$}

... la cité de Quinsai gir'environ.C.

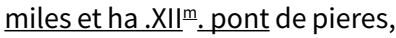
et por chascun de cesti pont, ou por la greingnor partie, poroit bien passer une (grant) nes por desout sun arche, et por les autres porroient passer mesn idre nes. Et nulz se face mervoille se il ha tant de pont, por ce qe je vos di qe ceste ville est toute en eve et est environcés de eve, et por ce convient qe maint pont hi aie por aler por toute la ville
... civitas Qinsay in circuitu suo girat miliaria çentum tere, et habet duodecim milia pontium, pro maiori parte de lapidibus, quia aliqui de legnamine sunt constructi; sub singulis quorum, vel pro maiori parte, transiret navis una, sub arcu, grandis et magna, per alios vero possent transire minores. Et nullus miretur si tot sunt ibi pontes: nam civitas tota est sita in aquis, et circundata aquis; et ideo necesse est ut sint ibi tot pontes ad hoc ut gentes possint per civitatem quacumque pertransire

\begin{abstract}
R II 68
Questa città, per commune opinione, ha di circuito cento miglia, perché le strade et canali di quella sono molto larghi et ampli; poi vi sono piazze dove fanno mercato [...] Et è fama che vi siano dodicimila ponti, fra grandi et piccioli: ma quelli che sono fatti sopra i canali maestri et la strada principale sono stà voltati tanto alti e con tanto magisterio [<VB?] che una nave vi puol passare di sotto senza albero $[<\mathrm{P}]$; et nondimeno vi passano sopra carrette et cavalli, talmente sono accommodate piane le strade con l'altezza. Et se non vi fussero in tanto numero non si potria andar da un luogo all'altro $[<\mathrm{P}]$

Dall'altro canto della città v'è una fossa...
\end{abstract}

25 Ramusio si attiene solitamente all'ordo di uno dei suoi modelli; gli spostamenti di pericopi sicuramente attribuibili alla sua iniziativa sono piuttosto rari e di solito riguardano porzioni di testo contenute.

26 Qui e negli esempi successivi, in carattere sottolineato i segmenti comuni alle tre redazioni, in grassetto quelli che accomunano R-Z $\mathrm{Z}^{\mathrm{t}}$ escludendo P-VB, in corsivo gli unica di R. 


\section{(b) Il consumo della carne}

\section{F 15114}

Et si vos di qe les jens sunt ydres et sunt au Grant Kan, et ont monoie de carte.

Il menjuent tutes cars, et de chiens e de toutes autres brutes bestes et autres animaus que por ren dou munde nul cristienç de sa ne menjuerént

\section{$Z^{t} 85$ 28-9}

Gentes adorant ydolla, monetam habent de cartis et sunt sub dominio Magni Can.

Comedunt omnes carnes, canum et generaliter omnium animalium

\author{
R II 68 14-5 \\ Vi sono poi le beccarie, dove \\ ammazzano gli animali grossi, \\ come vitelli, buoi, capretti et \\ agnelli, le qual carni mangiano \\ gli huomini ricchi et gran maestri; \\ ma gli altri che sono di bassa \\ conditione non si astengono da \\ tutte l'altre sorti di carni immonde, \\ senza havervi alcun rispetto
}

\section{(c) Il palazzo dell'imperatore Song}

\section{F 151 28-31}

Et en ceste cité est le palais dou roi qe se fuï, qe seingnor estoit ou Mangi, qui est le plus biaus e le plus noble qe soit au monde; e vos en diviserai aucune cousse. Or sachiés qe le palais gire environ .X. miles et est murés cun autes mures, toutes as querriaus, et dedens as mures sunt maint biaus jardis con tuit les buens fruit qe home seust deviser. Il hi a maintes fontaines et plusors lac, la o il ‘a〉 maint buen peison. Et, eu mileu, est le palais mout grandissme et biaus. Il a une si gran sale et si belle, qe grandisme quantité des jens hi poroient demorer et menjuere a table. La sale est toute portraite et pointe a penture d'or, et hi a maintes estoires et maintes bestes et hosiaus et chevalers et dames, et a maintes mervoilles. Il est mout bielle viste a garder, car en toutes les murs et en toutes covreoure ne poroit l'en veoir che pintures a or. Et que voç en diroie? Sachiés qe je ne vos poroie deviser la gran nobelité de cesti palais, mes je voç en dirai brefmant et sommeemant tout la verité. ¿Sachiés` de voir qe cest palais a .XX. sales, toutes d'une grant et d'un paroil; et sunt bien si grant qe. $X^{m}$. homes hi poroient menuier a table aaisemant; et sunt toute pointe a ouvre d'or mout noblemant. Et si voç di qe ceste palais ha bien .M. canbres, ce sunt maison bielles et grant, e de dormir et de mengier. Les frut et les pesciere vos ai contés.

\section{$Z^{t} 85$ 49-57}

In ista quidem civitate Qinsay est regale palatium, quod fuerat regis Facfur, domini provincie Manci, quod est pulcrius et nobilius aliquo quod reperiatur in mundo; de cuius facturis dicemus. Circuit enim palatium istud bene miliaria sex de terra. Est itaque altis muris valatum, et intra muros sunt multa pulcra viridaria omnimodos producentia bonos fructus. Sunt et ibi pulcri fontes et lacus quam plures, in quibus inveniuntur in habundantia boni pisces. Et in medio istius muri est palatium, valde pulcrum et magnum. In eo est quedam magna salla magistra, in qua simul discumberent multe gentes. Sala ista depicta est tota aureis picturis, $\underline{\text { ubi }}$ sunt ystorie diverse, bestie, aves, milites et domine cum multis mirabilibus; quod est pulcer intuitus ad videndum, quoniam in toto muro et omnibus coperturis, non posunt videri nisi solummodo ystorie colorate auro et aliis coloribus delectabilibus et pulcris. Palatium istud multas habet salas omnes spatiosas et pulcras, depictas ad aurum et subtiliter laboratas.

Habet insuper cameras bene mille; et est mirabile quid esse istius palatii. 


\begin{abstract}
R II 68 75-85
Hor parleremo d'un bellissimo palazzo dove habitava il re Fanfur, li predecessori del qual fecero serrare un spatio di paese che circondava da dieci miglia con muri altissimi, et lo divisero in tre parti. In quella di mezzo s'entrava per una grandissima porta, dove trovavansi da un canto e dall'altro loggie a piè piano grandissime et larghissime, col coperchio sostentato da colonne, le quali erano dipinte et lavorate con oro et azzurri finissimi; in testa poi si vedeva la principale et maggior di tutte l'altre, similmente dipinta con le colonne dorate, et il solaro con bellissimi ornamenti d'oro, et d'intorno alle parieti erano dipinte l'historie di re passati, con grande artificio. Quivi ogni anno, in alcuni giorni dedicati alli suoi idoli, il re Fanfur soleva tenir corte et dar da mangiare alli principali signori, gran maestri et ricchi artefici della città di Quinsai: et ad un tratto vi sentavano a tavola commodamente sotto tutte dette loggie diecimila persone. Et questa corte durava dieci o dodici giorni, et era cosa stupenda et fuor d'ogni credenza [...] cadauno claustro o vero corte havea cinquanta camere con li suoi giardini, et in tutte queste camere vi stantiavano mille donzelle che 'I re teniva alli suoi servitii; qual andava alcune fiate, con la regina et con alcune delle dette, a sollazzo per il lago, sopra barche tutte coperte di seda, et ancho a visitar li tempii degl'idoli. Le altre due parti del detto serraglio erano partite in boschi, laghi et giardini bellissimi, piantati di arbori fruttiferi, dove erano serrati ogni sorte di animali, ciò̀ caprioli, daini, cervi, lepori, conigli: et quivi il re andava a piacere con le sue damigelle, parte in carretta et parte a cavallo [...] Tutta questa narratione mi fu detta da un ricchissimo mercatante di Quinsai, trovandomi in quella città, qual era molto vecchio et stato intrinseco familiar del re Fanfur, et sapeva tutta la vita sua et havea veduto detto palazzo in essere, nel qual volse lui condurmi
\end{abstract}

\title{
3 Conclusioni
}

Si può discutere, a questo punto, di alcune ipotesi.

In primo luogo, pare difficile credere che Ramusio, nei capitoli su Quinsai, abbia interpolato la lezione dei suoi relatori poliani con una fonte odeporica 'non poliana' e non altrimenti nota, dalla quale avrebbe desunto la gran mole di informazioni esclusive: cosa che, tra l'altro, sarebbe in contrasto con le proposizioni di metodo dichiarate nella dedicatoria a Fracastoro posta in limine all'edizione. ${ }^{27}$

Altrettanto improbabile è che abbia attinto, per questa sola sezione, a un'ulteriore, non identificata redazione del Devisement, della quale, peraltro, non si rinviene traccia nel resto del libro.

Rimane, se non ci si inganna, una sola possibilità, che ha se non altro il pregio di evitare la moltiplicazione degli enti: e cioè che uno dei manoscritti ( $\mathrm{Z}^{\mathrm{g}} \mathrm{P}$ VB L V) serviti a Ramusio per costruire la sua edizione testimoniasse, all'altezza del trattato su Quinsai, una lectio singularis: ovvero, un testo profondamente rielaborato nella struttura e ricchissimo di informazioni inedite.

$27 \ll \ldots$ cosa ragionevole ho giudicato di far venir in luce il suo libro, col mezo di diversi esemplari scritti già piú di dugento anni, a mio giudicio perfettamente corretto e di gran lunga molto più fidele di quello che fin ora si è letto» (Dedicatoria al Fracastoro, § 10, in Simion, Burgio 2015). 
Che l'esemplare in questione vada individuato nel codice Ghisi è, naturalmente, l'ipotesi meno onerosa, stante la spesso disorientante mouvance testuale riscontrabile nei testimoni direttamente o indirettamente inquadrabili entro la 'costellazione Z' (cf. § 1). Si può dunque supporre - tornando alla proposta formulata in coda al $\S 1.2$ - che, a valle di $\beta^{\prime \prime \prime}$, uno degli antecedenti di $\mathrm{Z}^{\mathrm{g}}$ (o $\mathrm{Z}^{\mathrm{g}}$ stesso?) sia stato soggetto a una parziale riscrittura che avrebbe comportato l'innesto, su uno scheletro simile a quello di $\mathrm{Z}^{\mathrm{t}}$, di materiali testuali nuovi e soprattutto di prima mano. E del resto probabile che Marco Polo, o qualcuno dei suoi familiari, avesse conservato una grande quantità di informazioni su Quinsai sotto forma di appunti e ricordi, sfruttati solo parzialmente nella prima stesura del livre e successivamente rifusi in uno (o più?) esemplari $Z$, con o senza la sua supervisione. In alternativa - ma pare una soluzione meno economica - si dovrà credere che a Venezia, tra Tre e Quattrocento, circolassero altre raccolte odeporiche (zibaldoni di viaggiatori?) contenenti descrizioni di Quinsai, ${ }^{28}$ alcuni brani delle quali potrebbero essere stati interpolati nel testo di $\mathrm{Z}$ a integrazione del dettato poliano.

28 A questo proposito, nella seconda redazione della Relatio accolta nelle Navigationi di Ramusio, Odorico segnala di avere «ben trovate in Vinetia assai persone che vi [a Quinsai] sono state» (Milanesi 1978-88, 4: 311) 


\section{Tavole}

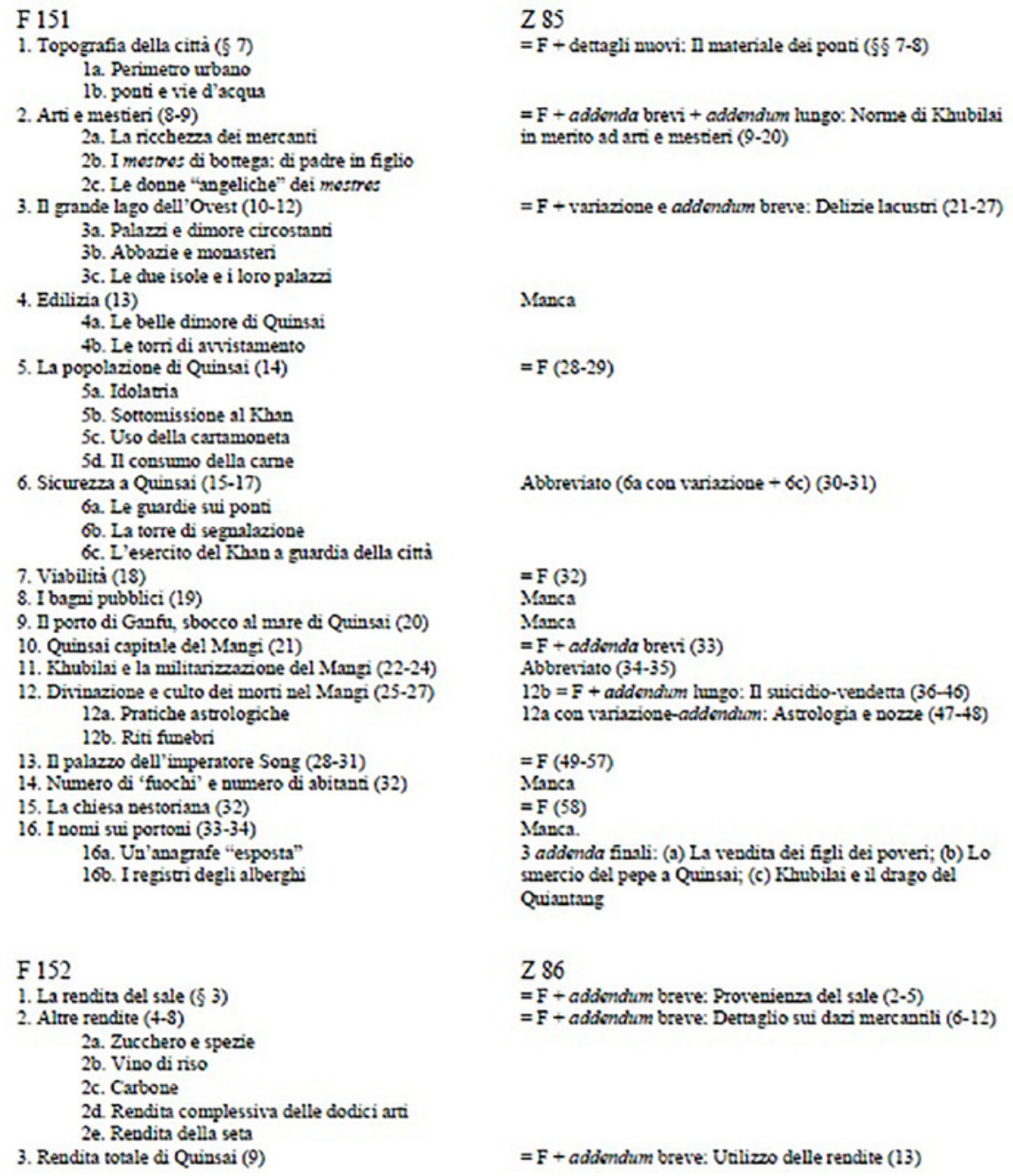

\section{F 151}

1. Topografia della città (§ 7$)$

1a. Perimetro urbano

1b. ponti e vie d'acqua

2. Arti e mestieri (8-9)

2a. La ricchezza dei mercanti

2b. I mesres di bortega: di padre in figlio

2c. Le donne "angeliche" dei mestres

3. I grande lago dell' Orest (10-12)

3a. Palazzi e dimore circostant

3b. Abbazie e monasteri

3c. Le due isole e $i$ loro palazzi

4. Edilizia (13)

43. Le belle dimore di Quinssi

4b. Le torri di atvistamento

5. La popolazione di Quinsai (14)

53. Idolatis

5b. Sottomissione al Khan

5c. Uso della cartamoneta

5d. Il consumo della came

6. Sicurezza a Quinsai (15-17)

6a. Le guardie sui ponti

66. La torre di segnalazione

$6 c$. L'esercito del Khan a guardia della citta

7. Viabilita (18)

8. I bagni pubblici (19)

9. I porto di Ganfu, sbocco al mare di Quinsai (20)

10. Quinssi capitale del Mangi (21)

11. Khubilai e la militarizzavione del Mangi (22-24)

12. Dixinazione e culto dei morti nel Mangi (25-27) 12a. Pratiche astrologiche

12b. Riti funebri

13. In palazzo dell'imperatore Song (28-31)

14. Numero di 'fuochi' e numero di abitant (32)

15. La chiesa nestoriana (32)

16. I nomi sui portoni (33-34)

16a. Un'anagrafe "esposta"

106. I registri degli alberghi

\section{F 152}

1. La rendita del sale (§ 3)

2. Altre rendite (4-8)
2a. Zucchero e spezie
2b. Vino di riso
2c. Carbone
2d. Rendita complessiva delle dodici arti
2e. Reodita della seta

3. Reudita totale di Quinsai (9)

\section{Z 85}

= F + dettagli nuovi: I materiale dei pouti (\$ई 7-\$)

= F + addenda brevi + addendion lungo: Norme di Khubilai in merito ad arti e mestieri $(9-20)$

= F + variazione e addendum breve: Delizie lacustri (21-27)

Manca

$=\mathrm{F}(28-29)$

Abbreviato (6a con variazione $+6 c)(30-31)$

$=\mathrm{F}$ (32)

Manca

Manca

$=\mathrm{F}+$ addenda brevi (33)

Abbretiato (34-35)

$12 \mathrm{~b}=\mathrm{F}+$ addendum lungo: I suicidio-vendetta (36-46)

12 a con variazione-addendion: Astrologia e nozze $(47-48)$

$=\mathrm{F}(49-57)$

Manca

$=\mathrm{F}(58)$

Manca.

3 addenda finali: (a) La vendita dei figli dei poveri; (b) Lo smercio del pepe a Quinsai; (c) Khublai e Il drago del Quiantang

Z 86

=F + addondum breve: Provenienza del sale (2-5)

= F + adicndum breve: Dettaglio sui dazi mercancli (6-12)

=F + addondum breve: Utilizzo delle reodite (13)

Tavola 1. Sinossi F 151-2 / Z 85-6 


\section{RII $6 \mathrm{~S}$}

1. Topografia della citra + addonda:

(a) Anpiezza di canali e strade: (o) Lazo, fumse, canali navigabiit: (c) Strutara dei pont: (d) Canslizazioni e govemo delle acoue: (e) Merci, meecanti, mercati (ingloba 5d. Il consumo della carne, nelaborato. e S. I

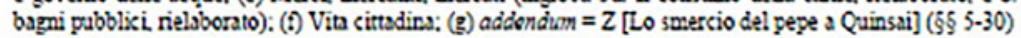

5a. Idolatria (31)

5c. Uso della caramoneta (31)

addendum lungo: Le vesti di seta (31)

2. Arti e mestieri, con addenda brevi $=Z(32-35)$

42. Le belle dimore di Quinsai (36)

addendion lungo: Mansuerudine degli abitanti di Quinsai (37.41)

3. I lago di Mezzogiorno + addendum lungo, con elementi $=Z$ [Delizie lacustri] (42-48)

7. Viateliti - addendum lungo: Il traffico stradale a Quinswi (49-52)

12. Dixinszione e culto dei morti nel Mangi con elementi $=Z$ [Astrologis e nozze] (53-56)

4b. Le torri di avvistamento (57)

6a. Le guardie sui ponti - addenda: (a) La segnalazione delle ore e il coprifuoco: (0) Ricovero delle merci in caso di inserdio (<VB) (58-63)

6c. L'esercito del Khan a guardia della citti (64)

6b. La torre di segaslazione (65)

10. Quinsai capitale del Mangi. con addenda brevi $=Z(66-68)$

11. Khublas e la militarizariose del Mangi + addendum lungo: I Tarari e il reciutamento dell'esercito nei docmini cinesi (69-74)

13. Il pulazzo dell' imperatore Sung, nelaborato con adienda (75.86)

9. Il porto di Ganfu, sbocco al mere di Quinssi + adienda brevi (87.88)

14. Numero di "fuochi" e numero di abitanti (\$9)

15. La chiesa nestoriana ( 89 )

16. I portoni della citta come registri d' anugrafe (90-91)

Addondum = Z: La vendita dei figli dei poveri (92)

Tavola 2. R II 68: struttura e contenuti 